\title{
La forme être en train de comme éclairage de la fonction de l'imparfait
}

\author{
Lidia LEBAS-FRACZAK \\ Université de Clermont-Ferrand II, LRL
}

\section{Introduction}

La description de l'imparfait, dans ses emplois «passés », s'étant toujours principalement faite en opposition au passé simple/passé composé, nous pensons qu'il est intéressant d'élargir le système d'oppositions en y incluant la forme être en train de (à l'imparfait) et en la confrontant, entre autres, aux critères aspectuels et anaphoriques.

Les grammaires utilisent souvent cette forme pour définir ou illustrer le sens d'énoncés à l'imparfait. Par exemple, dans la Grammaire Progressive du Français (Niveau avancé) ${ }^{1}$, destinée aux apprenants étrangers, nous trouvons une règle selon laquelle «l'imparfait indique que l'action est montrée en train de se faire dans le passé ». De manière identique, Le Bon Usage de Grevisse informe que « [l'imparfait] montre [un] fait en train de se dérouler ». Les linguistes se servent aussi parfois de cette forme pour gloser des exemples avec l'imparfait, là où l'imparfait est censé donner clairement une représentation du procès « dans son cours », comme le fait, par exemple, J. Bres (2005 : 2). De même, selon A. Molendijk, l'énoncé Il poussait trop loin la plaisanterie « renvoie à une situation qui est en train de se dérouler à un moment donné du passé : le fait rapporté a déjà commencé avant ce moment, et n'est pas encore parvenu à son terme (à ce moment) » (1985 : 79).

La valeur habituellement attribuée à la forme être en train de ressemble à celle de l'imparfait, faisant intervenir la notion de «déroulement». En retraçant l'évolution sémantique de être en train de, D. T. Do-Hurinville (2007) observe que depuis le milieu du XIX ${ }^{\mathrm{e}}$ siècle cette forme véhicule le sens de «déroulement d'une action en cours », ce qui représente l'aspect «progressif». Selon la définition de A. Borillo (2006), la fonction des « auxiliaires aspectuels adnominaux» (tels que être en train de) consiste à présenter le procès sous l'angle de son déroulement interne, c'est-à-dire à marquer l'aspect interne de la situation.

Malgré cette apparente ressemblance de valeurs, lorsqu'on observe de plus près des exemples attestés, on remarque que les deux formes ne sont pas interchangeables, même dans les contextes « canoniques » passés. Un test

1 M. Boularès et J.-L. Frérot, CLE International, 1997. 
nous suffira pour l'instant à le démontrer : le moteur de recherche Google nous a permis de constater la présence sur Internet de milliers d'occurrences de la séquence rien ne se passait et de la séquence il pleuvait, et la quasiabsence de leurs équivalents avec en train de (? rien n'était en train de se passer ; ? il était en train de pleuvoir). Comme nous l'expliquerons plus loin, c'est la nature du sujet, empêchant sa « focalisation », qui en est responsable.

\section{Critères sémantiques : sens « aspectuels » et « non aspectuels»}

Si une différence de valeur entre les deux formes existe, ce qu'on doit supposer comme pour toutes formes différentes, il convient de se demander en quels termes elle peut être définie. Bien que ce qui ressort des descriptions citées plus haut soit une ressemblance aspectuelle, on peut entreprendre d'affiner le critère aspectuel en vue d'une différenciation. En effet, alors qu'on utilise le terme "imperfectif » pour qualifier l'aspect lié à l'imparfait, la forme être en train de est associée, quant à elle, à l'aspect «progressif », même si, comme le note C. D. Push, «la distinction notionnelle et terminologique entre l'aspect imperfectif et l'aspect progressif n'est pas toujours bien respectée » $(2003: 496)$. Selon B. Comrie (1976), l'aspect imperfectif est une catégorie aspectuelle plus générale, plus abstraite que le progressif. Cependant, comme le lui reprochent certains auteurs (ex. Bybee, Perkins \& Pagliuca 1994, Push 2003), les définitions qu'il fournit de l'aspect imperfectif et de ses sous-catégories ne sont pas suffisamment précises; ainsi, l'imperfectif est censé correspondre à une situation envisagée de l'intérieur («viewing a situation from within », op. cit. : 23) et le progressif à la description d'une situation en cours de développement au moment référé (« situation in progress », op. cit. : 33). En effet, la distinction en ces termes n'est pas suffisante, dans la mesure où la caractéristique attribuée au progressif n'est pas incompatible avec l'imperfectif français (l'imparfait).

Comrie complète la description du progressif par une autre caractéristique : «sens non statif» («nonstative meaning», op. cit. : 35). Selon D. T. Do-Hurinville, cette caractéristique, qu'il formule comme « [+ dynamique]», constitue «l'invariant sémantique de train et de être en train de ». L'auteur ajoute que cela « explique pourquoi, de nos jours, cette périphrase est compatible avec les procès dynamiques, alors qu'elle accepte difficilement les procès non dynamiques» (2007:33). L. Mortier, en analysant les contraintes distributionnelles de la forme en question, remarque également qu'elle « se fait le plus facilement suivre des verbes exprimant des activités » (2005: 89). Cependant, c'est une tendance plutôt qu'une véritable contrainte, étant donné la possibilité des emplois comme ceux-ci :

(1) Cadin s'aperçoit également que Bernard Thiraud, la victime de l'enquête dont il est chargé, a été tué vingt ans après pour les mêmes raisons, parce 
qu'il était en train de comprendre l'importance des découvertes de son père. (http://jeunet.univ-lille3.fr/auteurs/daeninckx02/analyse.htm)

C'est indéniable, il était en train de tomber amoureux.

(http://www.inlibroveritas.net/lire/oeuvre12292-chapitre57299.html)

L. Mortier fait remarquer l'importance du caractère "agentif» (ainsi que « animé ») du sujet et note que « la compatibilité éventuelle d'être en train de INF avec les verbes psychologiques semble dépendre [...] de la mesure dans laquelle le verbe exprime une activité et le sujet est agentif» $(2005: 89)$. Cette observation peut probablement s'appliquer à l'exemple (1), car on imagine que le fait de comprendre est le résultat d'une recherche active de la part du sujet, mais pas vraiment à l'exemple (2), où le procès de tomber amoureux ne semble pas être envisagé comme étant contrôlé par le sujet ${ }^{2}$.

Bien qu'on ne puisse pas réfuter la préférence de la forme être en train de pour certains types de sujets et pour certains types de verbes, et qu'il faille en tenir compte, ces caractéristiques ne peuvent pas constituer des traits distinctifs par rapport à l'imparfait «simple», car elles ne sont pas incompatibles avec lui.

Bybee, Perkins \& Pagliuca (1994) préconisent pour les «périphrases progressives » de différentes langues (comme la forme anglaise be + -ing) une valeur intégrant à la fois un sens «aspectuel» ${ }^{3}$ et un sens «non aspectuel» apporté par les entités lexicales d'origine. Ce deuxième sens consiste à « localiser un agent comme étant au milieu d'une activité (« to give the location of an agent as in the midst of an activity », op cit. : 133), d'où la compatibilité avec les verbes «dynamiques ». Ainsi, le progressif n'est pas différencié de l'imperfectif en termes purement aspectuels, ce que nous avons également vu chez Comrie, avec le sens «non statif». Mais, si l'on considère, en suivant Bybee et al., que le progressif possède, outre sa caractéristique aspectuelle, la caractéristique sémantique préconisée, on continue à avoir du mal à différencier les valeurs de l'imparfait et de être en train de dans les énoncés comme (3) et (4), alors que la différence est plutôt sensible :

(3) Je suis allé chez Bob en emportant une bouteille. Quand je suis arrivé, Annie était en train de casser la vaisselle. En me voyant, elle a gardé un saladier soulevé au-dessus de sa tête, il y avait pas mal de débris sur le sol. (Frantext : Ph. Djian, $37^{\circ} 2$ le matin)

2 Certains analystes (ex. Fuchs et Léonard 1979, Leeman 2003) considèrent que l'emploi d'un verbe «statif», comme haïr ou comprendre, avec la forme être en train de, donne le sens " inchoatif».

3 «Progressive views an action as ongoing at reference time» (op. cit. : 126). 
(4) Lorsque Louis entra dans le salon, les enfants jouaient tandis que Céline faisait la vaisselle du petit déjeuner. "Vous avez déjà déjeuné !» Marmonna-t-il un peu déçu. (http://eveil.plumes.free.fr/16.htm)

En effet, pourrait-on affirmer que si le sujet Annie est « localisé comme étant au milieu de l'activité » en (3), cela n'est pas le cas pour Céline en (4) ?

Bybee et al. évoquent la nature différente, avec une « force adjectivale » (fonction de «caractérisation »), de la forme équivalente à be + -ing en ancien anglais ${ }^{4}$, mais considèrent que cette ancienne structure a disparu et que le progressif moderne a évolué à partir d'une structure locative. Quoi qu'il en soit, l'idée de «caractérisation » nous semble plus appropriée que celle de «localisation» pour parler des fonctions contemporaines des « progressifs » français et anglais (fonctions qui ne doivent pas pour autant être considérées comme identiques), ainsi que de la fonction de l'imparfait. La différence entre les deux formes françaises réside, selon nous, dans la portée de cette «caractérisation». En anticipant sur l'analyse que nous détaillerons plus loin, nous pouvons dire que dans l'exemple (3) la caractérisation porte sur le sujet (Annie), qui est donc l'élément focalisé, alors que dans (4) la caractérisation porte sur la situation trouvée par Louis au salon et à la cuisine, celle de «l'après petit déjeuner », ce qui fait que le sujet (Céline, de même que les enfants) n'est pas focalisé ici.

Bybee et al. précisent que le sens «locatif» originel des formes progressives tend vers le sens «d'implication du sujet dans l'activité ». On pourrait probablement considérer que le sujet dans l'exemple (3) est plus « impliqué dans l'activité » que celui dans l'exemple (4), du fait du caractère moins routinier de l'activité casser la vaisselle que de celle de faire la vaisselle. Mais il serait difficile de défendre le sens «d'implication » dans tous les cas, comme dans l'exemple suivant :

$$
\begin{aligned}
& \text { - Attendez, la fille, Aïcha, le modèle, vous l'avez vue ? insista Rovère, } \\
& \text { soudain troublé. } \\
& \text { - Je viens de vous le dire! Quand je suis arrivé chez Martha, elle était en } \\
& \text { train de poser. Une belle fille, d'ailleurs, une belle fille. (Frantext: } \\
& \text { T. Jonquet, Les Orpailleurs) }
\end{aligned}
$$

En effet, l'activité de poser en tant que modèle, en suivant les consignes du peintre ou du photographe, pourrait difficilement être considérée comme « impliquant le sujet » à un degré plus grand que, par exemple, l'activité de faire la vaisselle (à l'imparfait), comme en (4). Il nous semble que remplacer être en train de par l'imparfait dans l'exemple (5) ne changerait rien par

$4 \quad$ «It used a form of 'be' and the participle with adjectival force. It expressed a habitual or characterizing state, not active involvement in an activity.» (op. cit. : 135) 
rapport à «l'implication du sujet dans l'activité »; en revanche, cela perturberait la cohérence de cette partie du dialogue, qui se focalise sur le sujet Ä̈cha et non sur la «situation» (chez Martha) ni sur le «fait» de poser. Quant à l'impression «d'implication du sujet dans l'activité », nous la voyons comme un effet de sens possible lié à la focalisation du sujet, c'est-àdire à l'attention que lui porte le locuteur en le caractérisant par un prédicat à la forme être en train de.

Nous concluons de cette partie de l'analyse qu'une opposition entre l'imparfait simple et la forme être en train de à l'imparfait ne se laisse pas caractériser en termes aspectuels. Les différents sens «non aspectuels» attribués par certains auteurs aux formes dites progressives ne s'avèrent pas opératoires non plus pour effectuer une telle opposition.

\section{Critère anaphorique}

L’imparfait est aussi régulièrement analysé en termes "anaphoriques », même si, comme l'a observé G. Kleiber, «à assister au regain de la thèse imperfective dans les travaux récents sur l'imparfait, il semble bien que la saison n'est plus aux temps anaphoriques »(2003:8). Il convient donc de prendre en compte ce critère dans le cadre de l'opposition qui nous intéresse.

Selon l'approche anaphorique, l'imparfait est moins « autonome » que les formes perfectives car il a besoin d'être lié à un antécédent fourni par le contexte. Cet antécédent peut être vu comme étant de nature «temporelle » (cf. par exemple, Houweling 1982, Tasmowski-De Ryck 1985) ou bien « situationnelle» comme le préconise G. Kleiber, pour qui «l'imparfait indique une continuité avec une situation saillante, c'est-à-dire déjà introduite dans la mémoire immédiate en vigueur au moment de l'emploi de l'imparfait » (2003: 18).

On constate rapidement, en comparant des exemples comme (3) et (4) plus haut, que le critère anaphorique, qu'il soit de nature « temporelle » ou « situationnelle », n'est pas pertinent pour distinguer la forme être en train de (à l'imparfait) de la forme de l'imparfait simple. En effet, si on peut assigner à la description à l'imparfait en (4) un antécédent « temporel» (le moment où Louis entra dans le salon) ou «situationnel» (la situation qu'il trouve), la même opération est possible pour la description avec être en train de en (3) ainsi qu'en (5). Une valeur anaphorique apparaît aussi pour cette forme, de manière très nette, dans l'exemple ci-dessous, où elle accompagne la reprise du procès fixer :

(6) Vous avez peur pour vous ou bien pour ce que peut ouvrir cette clef ? Elle m'a fixé. Je me suis dit que je venais de marquer un point. Mais je me gourais complètement. Elle était en train de me fixer comme si je n'existais plus. (Frantext : J.-B. Pouly, La Clef des mensonges) 
Cela ne veut pas dire pour autant que l'anaphore constitue une caractéristique stable des descriptions avec être en train de. Ceci est également avancé au sujet de l'imparfait par certains auteurs, critiques de l'approche anaphorique (ex. Wilmet 1996, Desclés 2000, Bres 2007). Ainsi, on peut considérer que dans l'exemple (7) ci-dessous le «moment» et la «situation» de référence sont indiqués à l'aide du prédicat à la forme être en train de, et que c'est lui qui fournit donc un ancrage spatio-temporel au prédicat au passé simple, et non pas l'inverse.

(7) Jérôme Seignelay était en train de travailler sur un thème grec dans l'étude de Henri-IV lorsqu'il fut appelé au parloir. (Frantext : J. d'Ormesson, Le Bonheur à San Miniato)

Il est pertinent de faire remarquer que la forme « progressive » anglaise, be + -ing, est caractérisée par H. Adamczewski (1982) en termes anaphoriques (ainsi qu'énonciatifs) : cette forme servirait à reprendre une information déjà connue afin que l'énonciateur puisse la prendre en charge, ou produire un commentaire. L'auteur s'oppose avec cette conception à l'approche «traditionnelle», qui met en œuvre les notions telles que " durée », " aspect» et " forme progressive », en lui reprochant de confondre les plans linguistique et extralinguistique. Sa position trouve un large écho chez les anglicistes en France. F. Lachaux y fait référence, en comparant les formes anglaise et française et en affirmant que « les marqueurs be + -ing / être en train de signalent que la notion verbale est préconstruite, l'énoncé étant à interpréter en relation avec le «contexte-avant»(2005: 127). Elle retient également l'idée de «commentaire», sur laquelle nous reviendrons plus loin.

Comme nous l'avons signalé plus haut, un lien systématique entre la nature anaphorique du prédicat et la forme être en train de ne nous paraît pas certain. Mais ce qui est plus important est que le critère anaphorique n'est pas, tout comme le critère aspectuel, pertinent pour définir cette forme en opposition à l'imparfait, qui est, lui aussi, parfois associé au phénomène de l'anaphore.

\section{Analyses non aspectuels de la forme être en train de}

J.-J. Franckel (1989) fournit une analyse «énonciative » de la forme être en train de, s'inscrivant dans la théorie des opérations énonciatives de A. Culioli. Selon la définition proposée, la forme relève de l'opération de « différenciation » (qui s'oppose à l'opération « d'identification ») et signale un décalage entre le «perçu» (ou ce qui est «actualisé ») et le « représenté » (ou ce qui « devrait être »). Les différentes valeurs spécifiques qui apparaissent dans les énoncés varient selon la nature du décalage qui s'établit. Ce décalage peut être «temporel», entre ce qui est actualisé et ce 
qui est visé ou attendu ; c'est la «valeur classique de procès en cours » (ex. Le gâteau est en train de cuire, qui veut dire que le gâteau n'est pas encore cuit). Le décalage peut se matérialiser comme un rejet ou une démarcation par rapport à un autre procès, qui constitue un objectif construit par un tiers sujet (ex. Ne fais pas de bruit, il est en train de dormir). Il peut également se fonder sur une " altérité intersubjective », en apportant l'effet d'appréciation négative (ex. Qu'est-ce que tu es en train de faire? ; Il est en train de lire de travers).

L'analyse de J.-J. Franckel est nourrie d'exemples avec être en train de au présent. La question qui se pose est donc de savoir si le trait de « décalage » (ou de « différenciation ») pourrait être valable pour caractériser être en train de au passé et pour distinguer cette forme de l'imparfait simple. Si l'on compare par ce prisme les exemples (3) et (4), on peut avancer que la proposition Annie était en train de casser la vaisselle met en œuvre une sorte de décalage intersubjectif entre ce que fait le sujet et ce qui est attendu, ou jugé normal, par l'énonciateur-narrateur. Une telle interprétation paraît plausible étant donné le type de l'activité en question, pouvant être considérée comme violente et inhabituelle. Cet effet ne se manifeste pas dans l'exemple (4) à l'imparfait: Céline faisait la vaisselle, où l'activité en question n'a a priori rien de critiquable. L'exemple (5), avec elle était en train de poser, ne semble pas, en revanche, appuyer l'analyse de J.-J. Franckel. Il ne peut s'agir de décalage entre le stade « actuel» du procès et le stade final anticipé ou visé, car il serait étrange d'attribuer à l'énonciateur une attente quant au résultat ou but de poser. Il est difficile également d'y voir une « altérité intersubjective », car il s'agit plutôt d'une simple précision concernant le sujet. L'exemple (7) (Jérôme Seignelay était en train de travailler sur un thème grec) serait encore plus difficile à analyser en terme de « décalage ».

La définition de la valeur de la forme être en train de (au présent et à l'imparfait) proposée par F. Lachaux dépasse, elle aussi, le sens de « déroulement d'une action en cours ». L'auteur observe qu' « un énonciateur n'a pas recours à être en train de à chaque fois qu'il mentionne une activité en cours » et, d'autre part, qu' « il arrive que l'énonciateur y ait recours alors que ladite 'activité' n'est pas en cours au moment d'énonciation » $(2005: 121)$. Elle considère que «les notions sémantiques de 'procès en cours', de 'déroulement' (...) relèvent des effets de sens que la périphrase est susceptible de produire, mais ne suffisent pas à elles seules à expliquer la fonction de être en train de » (op. cit. : 123). La fonction première de cette forme serait de marquer une «mise en relief » d'un fait «préconstruit», liée à une intention argumentative consistant à justifier "autre chose». Par exemple : "ils sont en train de réparer la pompe, 'donc' indisponibles / ou 'donc' il faut en déduire que le problème de la pompe est sérieux » (op. cit. : 122). Le « relief énonciatif» est une notion comparable au «commentaire 
énonciatif» de H. Adamczewski, mais enrichie sur le plan de la coénonciation (ou intersubjectivité) : la forme être en train de " apparaît pour corriger une première impression, pour rétablir une 'vérité', pour répondre à une mise en doute éventuelle, non par le biais d'une simple contradiction, mais par une rhétorique persuasive » (op. cit. : 137), et il est demandé au coénonciateur «d'accepter comme pertinente une relation prédicative a priori non évidente » $\left(o p\right.$. cit. : 138) ${ }^{5}$. Il y a là aussi une compatibilité avec la valeur préconisée par J.-J. Franckel dans la mesure où la «mise en relief » est accompagnée d'une certaine forme de «décalage intersubjectif», car elle consiste dans la «négation, a priori, de l'interprétation que pourrait faire le co-énonciateur» (op. cit. : 137).

La théorisation proposée par F. Lachaux est solidement construite et convaincante, au vu des exemples utilisés. Il nous semble, cependant, que la valeur argumentative-intersubjective préconisée ne se retrouve pas telle quelle dans tous les exemples de notre corpus (Frantext et Internet) avec la forme être en train de à l'imparfait. Dans l'exemple (5) plus haut, l'information elle était en train de poser véhicule bien une information qu'on peut considérer comme " non évidente » pour le co-énonciateur, relativement au fait que l'énonciateur ait vu le sujet (la fille, Ä̈cha, le modèle) : " elle était en train de poser, 'donc' elle était là, 'donc' je l'ai vue ». La valeur en question est plus difficile à défendre, en revanche, dans l'exemple (7); on ne voit pas pourquoi le fait que Jérôme Seignelay était en train de travailler sur un thème grec serait mis en relief, ou considéré comme non évident, ou important pour justifier autre chose.

On peut essayer de tester cette approche sur encore un autre exemple :

(8) C'était un dimanche et Caroline, privée de Fafa, était en train de donner à goûter aux petits. Soudain alarmée par les cris suraigus des aînés qui jouaient dans le jardin, elle avait prié Sylvain d'aller voir ce qui se passait. (Frantext : G. Dormann, La Petite main)

On peut fournir l'interprétation suivante en termes de F. Lachaux : «puisque Caroline (...) était en train de donner à goûter aux petits, elle ne savait pas ce que faisaient les aînés ». Mais peut-on vraiment considérer qu'il s'agit là d'une information "non évidente » pour le récepteur, sujette à un éventuel doute ou contredisant une éventuelle "première impression"? $\mathrm{Si}$ on remplace être en train de par l'imparfait (Caroline donnait à goûter aux petits), on remarque, effectivement, la disparition d'une certaine «mise en relief » de l'information, mais nous l'expliquons par la «dé-focalisation » de la relation prédicative, qui fait que ni le sujet ni le prédicat n'est au centre

5 Il faut préciser que la valeur proposée par F. Lachaux est spécifique à la forme française être en train de, qui est, selon elle, «beaucoup plus marquée pragmatiquement » que la forme anglaise be + -ing (op cit. : 138). 
d'intérêt, car la proposition (à l'imparfait) ne sert alors qu'à caractériser la situation. Avec la forme être en train de, en revanche, la focalisation est sur Caroline, ce qui reste en cohérence avec le reste du fragment (et ce qui fait que, intuitivement, on juge l'emploi de cette forme plus adapté ici que celui de l'imparfait). Une telle explication se montre également efficace pour l'exemple (7) : avec la forme être en train de, on marque la focalisation sur le sujet, Jérôme Seignelay, alors que le paragraphe précédent parle de son père ${ }^{6}$.

L'idée de «focalisation sur le sujet» (ou de «caractérisation du sujet »), que nous associons à la forme être en train de à l'imparfait, se retrouve dans la description proposée par F. Lachaux, bien que cette idée soit subordonnée à la caractéristique de «mise en relief pour justifier autre chose ». L'auteur note en effet que, en employant cette forme, « l'énonciateur 'dit' quelque chose à propos du référent du sujet» (op. cit. : 134). Cette idée est développée à un autre moment de la façon suivante : "L'énonciateur présente le référent du sujet dans une situation qu'il met en avant (...), le référent du sujet n'est plus en position d'agent mais objet d'un discours (voilà ce que moi énonciateur j'“en' dis) » (op. cit. : 121), ou encore ainsi : « il n'est pas question d'action, mais d'état, plus précisément de la situation du référent du sujet » (op. cit. : 122). F. Lachaux rappelle également la formulation de H. Adamczewski (1982:61) selon laquelle avec la forme anglaise be + -ing (à la différence de la forme simple), l'énoncé «est orienté vers le sujet grammatical » (op. cit. : 125). C'est donc cette idée «d'orientation vers le sujet », ou du sujet devenu « objet d'un discours », qui nous paraît centrale et que nous proposons de retenir pour décrire la fonction de la forme être en train de en opposition à l'imparfait et au passé composé (et passé simple). Et concernant l'impression de «mise en relief» et l'impression d'importance pour faire comprendre «autre chose», elles s'expliqueraient, là où elles apparaissent, par le fait que si le locuteur prend la peine d'énoncer quelque chose sur le sujet (non nécessairement à l'aide de la forme être en train de d'ailleurs), cette information a forcément une pertinence pour le propos général, ou la visée argumentative, c'est-à-dire pour « autre chose » que l'information en elle-même.

\section{Analyse selon le critère pragmatique de « (dé-)focalisation »}

Ainsi, la fonction d'un prédicat à la forme être en train de est de fournir une information sur le sujet, de le caractériser, tout en montrant que c'est le sujet qui est «l'objet du récit» et qui est donc focalisé, et non le prédicat luimême ou une autre partie de l'unité discursive concernée. Cette valeur est d'ailleurs plutôt transparente au vu de la constitution de la forme en question, notamment la présence du verbe être : on peut dire qu'il s'agit d'un cas

6 J. d'Ormesson, Le bonheur à San Miniato, Paris : J.C. Lattès, 1987, p. 235. 
particulier de construction «attributive», sachant qu'une telle construction permet la caractérisation du sujet. La caractérisation se fait ici à l'aide d'un verbe à l'infinitif introduit par en train de et non, par exemple, à l'aide d'une expression adjectivale, comme dans elle est / était jolie, ou nominale, comme dans elle est / était médecin. Selon les grammaires, un verbe à l'infinitif peut d'ailleurs fonctionner comme attribut, par exemple dans Cette pièce est à repeindre.

Certains verbes, caractérisant le sujet par nature (y compris ceux qu'on qualifie «d'attributifs », comme paraitre, devenir, etc.), n'ont pas besoin de la forme être en train de pour orienter la focalisation sur le sujet. C'est le cas des verbes plaire et aimer, qu'on observe à l'imparfait dans l'exemple (9) cidessous, et qui permettent de caractériser, respectivement, le sujet $i l$ et le sujet ma grand-mère.

(9) Oui ! Mais il plaisait aux dames ! Ma tante, par exemple ! (...) Ma grandmère, en revanche, aimait beaucoup Robert Mitchum.

(http://dvdtoile.com/Thread.php?33827)

Ainsi, on peut rendre compte de la contrainte distributionnelle consistant dans l'incompatibilité de la forme être en train de avec certains verbes («statifs» ou "psychologiques») et dans sa compatibilité avec les verbes «d'action», que nous qualifierions plutôt, dans ce cadre, de «non caractérisants » a priori. Ces derniers ont besoin de l'opérateur en train de, associé au verbe attributif être, pour pouvoir remplir la fonction de caractérisation du sujet.

Nous avons déjà illustré la fonction préconisée en comparant les exemples (3) et (4); nous l'avons fait également avec les exemples (5) et (7). Une analyse identique s'impose pour l'exemple (6), où la proposition elle était en train de me fixer comme si... fournit un commentaire sur le sujet, sur son attitude. On perçoit bien que c'est le sujet (elle) qui est au centre de l'attention dans cette partie du récit.

L'exemple (10) ci-dessous ressemble à (6) dans la mesure où il s'agit également de l'interprétation que fournit le locuteur-narrateur du comportement, de l'attitude du sujet, sur lequel se focalise le fragment.

Seulement, il a continué sur sa lancée. - Je vais me changer... J'ai juste le temps de me changer... à mon avis, il était en train de dérailler, éplucher une banane aurait été au-dessus de ses forces. (Frantext: Philippe Djian, $37^{\circ} 2$ le matin)

On retrouve donc, avec ces deux exemples, un type de caractérisation qu'on peut qualifier de "commentaire énonciatif» du fait de la subjectivité explicite. Dans l'exemple (11) ci-dessous, on est toujours dans le cas de la 
focalisation sur le sujet, mais la caractérisation se veut plus « objective», n'étant pas explicitement prise en charge par le locuteur-narrateur.

(11) Il retardait à cause d'eux le plus possible le moment de sa retraite et continuait à enseigner la géométrie, tenant un discours devenu presque incompréhensible, d'un incompréhensible encore aggravé du fait que tout ce qu'on enseignait ailleurs était en train de changer: car l'aube se levait des mathématiques Modernes. (Frantext : J. Roubaud, Mathématique : récit)

L'élément focalisé (caractérisé) correspond donc au sujet représenté par l'expression complexe tout ce qu'on enseignait ailleurs, et la caractéristique qui lui est associée à l'aide de l'expression était en train de correspond à changer. Le prédicat de la proposition suivante (introduite par car) est à l'imparfait car ce prédicat est « dé-focalisé »; la proposition sert à présenter la « situation » dans laquelle s'inscrit l'élément focalisé, c'est-à-dire tout ce qu'on enseignait ailleurs. L'emploi de l'imparfait dans cette dernière expression s'explique, à son tour, par le fait que la focalisation n'est pas au sein de cette relation prédicative (ce qui veut dire que ni le sujet on ni le prédicat enseigner... n'est focalisé); elle est «au service» du sujet (« complexe») qu'elle permet de constituer. Se confirme donc, dans cet exemple, la fonction « dé-focalisante » de l'imparfait.

Afin de vérifier l'hypothèse concernant la différence de portée de la caractérisation (et donc de la focalisation) entre l'imparfait simple et la forme être en train de à l'imparfait, nous avons étudié le comportement de ces formes avec un sujet "indéfini». L'idée étant qu'un sujet indéfini, $i l$, ou négatif, rien, par exemple, devrait être incompatible avec la forme être en train de, si cette forme marque effectivement la focalisation sur le sujet. Cela se confirme, grâce à une recherche sur Internet, déjà mentionnée en introduction, avec la quasi-absence de séquences telles que rien n'était en train de se passer et il était en train de pleuvoir (en discours direct au passé) ${ }^{7}$ alors qu'on trouve des milliers d'occurrences de rien ne se passait ou il pleuvait. En effet, l'emploi de l'imparfait avec un sujet indéfini ne pose pas de problème dans la mesure où l'objet de la caractérisation n'est pas le sujet mais quelque chose d'autre, en dehors de la relation prédicative (une situation, une journée...). Dans l'exemple (12) ci-dessous, il s'agit de caractériser un quartier d'une petite ville de Californie :

(12) Jamais rien ne se passait ici, dans ce quartier d'une petite ville de Californie. La vie, à force d'être calme, en devenait monotone.

(www.atelier-web.com/gladys/un_bien_etrange_voisin.htm)

Bien que L. Mortier affirme que « quant aux verbes impersonnels, il s'avère que seuls les verbes météorologiques sont compatibles avec l'aspect progressif » (2005: 89). 
Cette hypothèse permet donc de fournir une explication générale aux faits distributionnels observés par d'autres auteurs, dont nous avons parlé plus haut : la préférence de la forme être en train de pour les sujets de type « animé » et «agentif». S'agissant de caractériser le sujet, il est naturel qu'un sujet « purement grammatical » $i l$ ou un sujet «négatif » comme rien ne soient pas compatibles. À propos de ce dernier, il a également été observé (par L. Mortier, par exemple) que la forme être en train de ne s'accommode pas très bien de la négation. Cela est vrai pour une relation prédicative avec rien comme sujet, car comment pourrait-on vouloir caractériser quelque chose dont on nie l'existence ? En dehors de ce cas, il semble a priori étrange de caractériser quelqu'un avec une caractéristique "négative », par exemple il n'était pas en train de mourir, à moins que ce soit dans un contexte (qu'on peut considérer comme polyphonique) où le locuteur conteste une caractéristique présupposée, comme dans l'exemple suivant :

(13) C'était à cet endroit, devant la maison, une fois rentré chez lui qu'il convenait de mourir. Mais il n'était pas en train de mourir, il était vivant, le cœur battait, les yeux voyaient, le cerveau pensait.

(http://perso.orange.fr/jplanque/Retour_a_la_maison.htm)

Nous considérons donc que la forme être en train de permet de focaliser le sujet, alors que l'imparfait sert à «dé-focaliser» le prédicat. Cette conception de l'imparfait est très proche de celle proposée par O. Ducrot (1979) ${ }^{8}$, selon qui « la substitution à l'imparfait d'autres temps du passé (...) fait apparaître seulement une différence de point de vue, de perspective, de centre d'intérêt ${ }^{9}$ (op cit. : 1) et « on a l'impression que les événements présentés à l'imparfait ne constituent pas vraiment l'objet du récit» (op. cit. : 10). L'auteur précise que l'imparfait a la fonction de «transformer l'événement en qualité » (op. cit. : 3). L'élément qualifié par un prédicat à l'imparfait est un «thème temporel », qui est «soit une période du passé, soit, plus fréquemment, un objet ou événement considéré à l'intérieur d'une certaine période du passé » (op. cit.: 6). Les notions de "thème » et de «focus » sont proches ${ }^{10}$, mais il n'est pas utile, selon nous, de retenir le terme «temporel» ou celui de «période passée ». O. Ducrot se sert, entre autres, de l'exemple suivant:

Voir aussi (Anscombre 1992).

C'est nous qui soulignons.

«Le terme focus est souvent employé dans un sens proche de celui de centre (center) pour désigner l'objet privilégié d'une attitude ou d'un processus cognitif. C'est ainsi qu'on trouve dans les approches de pragmatique nonformelle les notions de focus of attention (Chafe 1974, Dryer 1996), focus of interest (Bolinger 1985), focus of empathy (Kuno 1977) ou focus of contrast (Chafe 1976).» (J.-M. Marandin, Sémanticlopédie) 
À midi, M. de Villeneuve entra. Nous étions dans le salon et y formions un tableau très agréable. M. Crimp se faisait peindre. M. de Saint Lambert lisait dans un coin. Je jouais aux échecs avec Mme d'Houdetot (...).

Selon lui, les énoncés à l'imparfait «visent à qualifier l'instant où a lieu l'entrée de M. de Villeneuve » et fournissent une "description statique du salon à un moment précis» (op. cit. : 13). En termes de «focalisation / caractérisation ", nous dirons qu'il ne s'agit pas ici de caractériser les différents sujets (ce qui serait le cas avec la forme être en train de) mais, comme c'est d'ailleurs explicitement indiqué dans le texte, le tableau qu'ils forment ensemble dans le salon. La focalisation n'est donc pas sur les sujets, ni sur les faits exprimés par les prédicats; on caractérise la «situation » (plutôt que « l'instant» que mentionne Ducrot).

Comme nous l'avons suggéré dans une analyse plus haut, la fonction de dé-focalisation du prédicat permet de «subordonner» (pragmatiquement) l'information véhiculée par une proposition à l'imparfait à une autre information qui est, elle, focalisée. Cela converge avec la nature «non autonome » de l'imparfait, postulée notamment par l'approche anaphorique, et avec l'idée « d'antécédent » pour un prédicat à l'imparfait, d'autant plus si l'on considère que cet antécédent est de nature « situationnelle» plutôt que (purement) «temporelle», comme dans (Kleiber 2003) ${ }^{11}$. En effet, il est naturel que soit présupposé (ou «antérieur») ce sur quoi porte une caractérisation (ici, celle effectuée à l'aide d'une proposition à l'imparfait). Mais, ce n'est pas cette présupposition (ou antériorité) de la situation qui dicte directement l'emploi de l'imparfait, comme le voudrait l'approche anaphorique, car cela reviendrait à définir cette forme par une simple contrainte discursive, sans lui attribuer de fonction propre.

En entreprenant de définir la fonction de l'imparfait (ainsi que de ces formes concurrentes), nous nous intéressons aux intentions du locuteur relatives d'une part au propos qu'il formule et d'autre part au fait d'orienter l'interprétation de l'interlocuteur. Cette fonction peut être cernée avec la question suivante: «qu'est-ce qui se trouve au centre du propos?». Une telle analyse pragmatique a également un avantage sur l'approche aspectuelle, car elle permet de remonter à la source du choix de la forme, en deçà des effets de sens. L'approche aspectuelle décrit, quant à elle, les effets de sens sans s'intéresser à leurs sources, puisqu'elle n'explique pas pourquoi tel ou tel «aspect» est (peut ou doit être) choisi dans un contexte donné, c'est-à-dire pourquoi un procès est envisagé «de l'intérieur» ou «de l'extérieur» (ou «globalement»), ou pourquoi le locuteur est censé

11 On notera que la théorie de O. Ducrot (1979) est aussi rapprochée de la thèse « anaphorique» par certains auteurs. O. Ducrot fait lui-même une rapide référence au "point de référence » de $\mathrm{H}$. Reichenbach en introduisant la notion de « thème temporel». 
s'intéresser ou non aux «limites » du procès. Les impressions aspectuelles trouvent une explication dans le cadre de notre conceptualisation. Ainsi, l'imperfectivité d'un prédicat (la non prise en compte des «limites»du procès, vue «partielle » ou « intérieure ») découle de la dé-focalisation du prédicat, c'est-à-dire que le procès ne nous intéresse pas en tant que tel, pour lui-même et avec tous ses attributs (ex. la durée), mais pour caractériser « autre chose ». La perfectivité (vue « entière » ou " globale ») d'un prédicat reflète, quant à elle, la focalisation sur le prédicat / procès, c'est-à-dire son intérêt en tant que tel et pour lui-même.

\section{Intégration du passé composé / passé simple}

Le choix de la forme du verbe se fait donc en fonction du rôle que le prédicat doit remplir dans le discours, en relation avec l'intention de focalisation (centre d'intérêt, objet du récit, objet de la caractérisation). Cette focalisation peut se placer à l'intérieur d'une relation prédicative donnée (soit sur le sujet soit sur le prédicat) ou bien à l'extérieur de la relation prédicative. Nous avons vu que l'imparfait dé-focalise le prédicat, qui peut ainsi servir à caractériser un autre élément, alors que la forme être en train de permet d'orienter la focalisation sur le sujet. Inclure le passé composé (et le passé simple) dans le jeu d'oppositions selon ce même critère revient à ajouter un troisième cas de figure : focalisation sur le prédicat ou, autrement dit, caractérisation d'un « fait » (ou " procès »).

Une comparaison s'impose avec les approches «textuelles », initiées par l'analyse de H. Weinreich (1973), qui a eu le mérite de déplacer le plan d'analyse de celui de la phrase et des réalités extra-linguistiques (ou « contenus du discours ») à celui du texte et de la communication ${ }^{12}$. On pourrait considérer que la focalisation du prédicat correspond à son appartenance au «premier plan » et que sa dé-focalisation revient à le placer en "arrière-plan». Cette correspondance rencontre cependant des limites, dans la mesure où la dé-focalisation d'un prédicat donné ne va pas toujours de pair avec la focalisation d'autre chose (notamment dans les emplois « modaux »), et que, s'il y a bien une focalisation ailleurs, elle n'est pas nécessairement exprimée par un verbe au passé simple ou au passé composé (ou par un verbe tout court), comme dans l'exemple (15) plus bas, où les éléments caractérisés (focalisés) sont les journées d'hier et d'aujourd'hui. En outre, la binarité de la division en premier plan et en arrière-plan ne permet pas l'intégration de la forme être en train de et la prise en compte de la focalisation du sujet.

12 Pour les critiques de cette approche, voir (Labelle 1987, Molendijk 1990, O’Kelly 1995). 
Afin d'illustrer la différence entre les formes de l'imparfait et du passé composé en termes de " (dé-)focalisation », nous allons comparer deux paires d'exemples : (15) et (16) à l'imparfait avec (17) et (18) au passé composé.

(15) Trop bizarre le changement de temps en Angleterre! hier il neigeait et aujourd'hui il fait un magnifique soleil et avec un gros pull on n'aurait presque pas besoin de manteau. (http://monptitmondeamoi.hautetfort.com/archive/2006/03/index.html)

D'une mère française et d'un père argentin, Rafaël Pividal vient d'un milieu modeste. En réalité, il est issu d'un milieu plutôt bourgeois, son père était avocat, sa mère, danseuse classique.

(http://fr.wikipedia.org/wiki/Rafaël_Pividal)

Dans l'exemple (15), la caractérisation ne porte pas sur le sujet, ce qui est d'autant plus évident qu'il s'agit d'un sujet «grammatical». Le prédicat n'est pas focalisé non plus car le fait de neiger n'est pas important en luimême, mais en tant qu'il permet de caractériser la journée d'hier (en comparaison avec la journée d'aujourd'hui) ; on pourrait même remplacer $i l$ neigeait par il faisait froid et humide, par exemple, ou par c'était l'hiver, sans changement notable du sens de l'énoncé. De même, dans l'exemple (16), le fait d'être avocat n'est pas important en tant que tel, mais en tant qu'il permet d'illustrer le milieu (plutôt bourgeois) de Rafaël Pividal.

(17) Il a neigé dans la nuit de lundi à mardi à la Réunion, phénomène très rare dans une île tropicale.

(http://www.liberation.fr/actualite/reuters/reuters_france/209643.FR.php?rss $=$ true)

(18) A l'origine, une carte d'identité perdue. Jacques Laurent est né à Paris, mais la production de l'extrait d'acte de naissance ne règle pas le problème. Son père a été avocat, son grand-père maternel officier de marine, son grandpère paternel président du conseil général de la Seine; ces professions et fonctions ne peuvent être exercées que par un Français mais cela ne suffit pas. (http://www.snes.edu/memos/g0/g0_t1132.htm)

Dans l'exemple (17), la caractérisation porte sur le «fait» exprimé par le prédicat : à la différence de (15) plus haut, on s'intéresse ici à l'événement de neiger pour lui-même, avec ses «attributs » : date et lieu; la raison de cet intérêt est claire grâce à la suite de l'énoncé. Dans (18), la focalisation sur les faits exprimés par les prédicats au passé composé est également rendue évidente par la suite de l'énoncé, où l'importance des professions qu'ils expriment se trouve explicitée.

Concernant la focalisation sur le fait (événement, action, état...) avec le passé composé, il est important de préciser que, en dehors de l'intérêt pour la nature même du fait (ex. que le métier de quelqu'un ait été avocat), l'intérêt peut porter plus particulièrement sur une autre caractéristique du fait: sa 
durée, son lieu, le moment de son occurrence, son résultat ou une autre particularité, comme l'illustrent les exemples plus bas. Ainsi, l'explication de la compatibilité du passé composé avec l'expression de la durée consiste à dire que si l'on s'intéresse à une caractéristique d'un fait (sa durée en l'occurrence), cela veut dire qu'on s'intéresse à ce fait lui-même ; le prédicat concerné se trouve donc focalisé. La dé-focalisation du prédicat comme fonction de l'imparfait explique pourquoi cette forme n'est pas associable à une expression de durée : si le fait (ou procès) n'est pas au centre d'intérêt, on ne s'intéressera pas à sa durée.

Nous analysons ci-dessous quelques exemples au passé composé :

$$
\begin{aligned}
& \text { James Grippando a été avocat pendant une dizaine d'années et se consacre } \\
& \text { aujourd'hui exclusivement à l'écriture. } \\
& \text { (http://www.bm-tence.fr/opac/index.php?lvl=publisher_see\&id=19) }
\end{aligned}
$$

L'intérêt pour le fait d'avoir été avocat, ainsi que pour sa durée, est sans doute motivé ici par l'intention de faire savoir que l'écriture n'a pas toujours été l'occupation unique de cet écrivain.

$$
\begin{aligned}
& \text { Il a suivi un parcours atypique, dans la mesure où il est devenu avocat } \\
& \text { relativement tard, à } 38 \text { ans. (http://mapage.noos.fr/mricard/associes.htm) }
\end{aligned}
$$

Ici, ce qui motive l'intérêt pour le fait en question est le moment, tardif, où il s'est produit dans la vie du sujet.

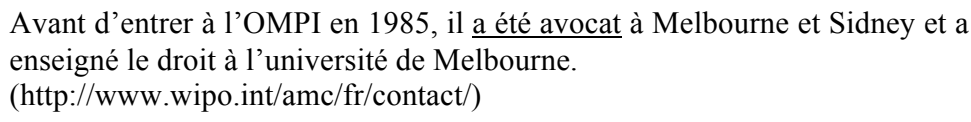

Avant d'entrer à l'OMPI en 1985, il a été avocat à Melbourne et Sidney et a enseigné le droit à l'université de Melbourne. (http://www.wipo.int/amc/fr/contact/)

C'est un exemple typique d'un discours biographique, où l'on s'intéresse aux faits de la vie d'une personne, leurs lieux, dates et durées.

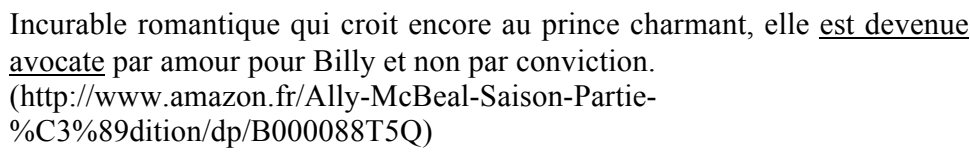
avocate par amour pour Billy et non par conviction. (http://www.amazon.fr/Ally-McBeal-Saison-Partie\%C3\%89dition/dp/B000088T5Q)

Dans cet exemple, la raison d'évoquer (et de focaliser) le fait de devenir avocate découle de l'intérêt qu'on porte à ce qui a motivé ce fait dans la vie du personnage.

Au sujet des exemples avec devenir, on pourrait considérer que la forme du passé composé est due à l'aspect «ponctuel» du procès. Mais il est possible, bien évidemment, d'employer ce verbe à l'imparfait, comme dans l'exemple (23), et il nous semble que ce n'est pas pour l'envisager « de l'intérieur » ou pour le présenter comme « étant en cours ». 
(23) Edgar Faure est né le 18 août 1908 à Béziers (Hérault). Il s'est fait remarquer très jeune puisque dès 1929, il devenait avocat à la cour de Paris. Parmi la très longue liste de ses éminentes fonctions, on retiendra celles de ministre et de président du Conseil.

(http://www.voixdujura.fr/archives/voir_archive.asp?archive=1756\&dossier $=\&$ chronologie $=$ oui\&page $=133$ )

Nous expliquons la différence en terme de la focalisation : alors que dans l'exemple (22) l'intérêt porte sur le fait lui-même de devenir avocat, cela n'est pas le cas dans l'exemple (23), où le fait de devenir avocat est mentionné pour illustrer le début de carrière précoce (dès 1929), qui est une information focalisée. Une analyse en termes de dé-focalisation du prédicat est valable, selon nous, pour tous les cas de ce qu'on appelle « imparfait de rupture » ou « narratif». Tout comme pour les emplois dits « modaux », que nous n'aborderons pas ici, par manque de place.

\section{Conclusion}

Ayant considéré que les descriptions d'ordre aspectuel ne s'avéraient pas efficaces pour réaliser une différenciation entre l'imparfait (simple) et la forme être en train de (à l'imparfait), pas d'avantage que le critère anaphorique, nous avons proposé un autre critère, de nature pragmatique, celui de "(dé-)focalisation ». Partant de l'idée que le prédicat possède par défaut un statut informatif central dans la phrase ${ }^{13}$, ce qui est compatible avec sa focalisation, on peut considérer que certaines formes servent à déplacer cette focalisation, ou à « dé-focaliser » le prédicat, au profit du sujet ou d'une autre information en dehors de la relation prédicative. Ce trait pragmatique, que nous situons en amont des effets «aspectuels» et « anaphoriques », permet d'expliquer la fonction de l'imparfait, c'est-à-dire la dé-focalisation du prédicat, en opposition à la forme être en train de, qui focalise le sujet, ainsi qu'aux formes du passé composé et du passé simple, qui maintiennent la focalisation sur le prédicat.

\section{Références}

Adamczewski, H. (1982). Grammaire linguistique de l'anglais, Paris : A. Colin.

Anscombre, J.-C. (1992). Imparfait et passé composé : des forts en thème / propos, Information grammaticale $55:$ 43-53.

13 Comme le rappelle L. Roussarie dans Sémanticlopédie, «certaines approches définissent le prédicat comme l'unité la plus informative de la phrase ». 
Bat-Zeev Shyldkrot, H.; Le Querler, N. (éds), (2005). Les Périphrases Verbales, Linguisticae Investigationes Supplementa 25, John Benjamins.

Borillo, A. (2006). Quelques formes lexicales de l'aspect adnominal en français, Cahiers de Grammaire 30 : 21-38.

Bres, J. (2005). L'imparfait : l'un et/ou le multiple? A propos des imparfaits « narratifs » et « d'hypothèses », Cahiers Chronos 14:1-32.

Bres, J. (2007). Sémantique de l'imparfait: dépasser l'aporie de la poule aspectuelle et de l'oeuf anaphorique? Éléments pour avancer, Cahiers Chronos $16: 23-46$.

Bybee, J. ; Perkins, R. ; Pagliuca, W. (1994). The Evolution of Grammar. Tense, aspect, and modality in the languages of the world, Chicago/London: University of Chicago Press.

Comrie, B. (1976). Aspect. An introduction to the study of verbal aspect and related problems, Cambridge: Cambridge University Press.

Desclés, J.-P. (2000). Imparfait narratif et imparfait de nouvel état, Colloque de Cracovie, Pologne, septembre 2000. (http://www.lalic.paris4.sorbonne.fr/PUBLICATIONS/communications. php?annee $=2000$ )

Do-Hurinville, D. T. (2007). Du substantif train à la locution adverbiale être en train et à la locution aspectuelle être en train de, L'Information Grammaticale $113: 32-39$.

Ducrot, O. (1979). L'imparfait en français, Linguistische Berichte 60 : 1-23.

Franckel, J.-J. (1989). Étude de quelques marqueurs aspectuels du français, Genève : Droz.

Fuchs, C.; Léonard, A.-M. (1979). Vers une théorie des aspects, Paris-La Haye-New York : Mouton et EHESS.

Houweling, F. (1982). Deictic and anaphoric tense morphemes, Journal of Italian Linguistics $7: 1-30$.

Kleiber, G. (2003). Entre les deux mon cour balance ou l'imparfait entre aspect et anaphore, Langue Française 138 : 8-19.

Labelle, M. (1987). L'utilisation des temps du passé dans les narrations françaises : Le Passé Composé, L'Imparfait et Le Présent Historique, Revue Romane, 22-1 : 3-29.

Lachaux, F. (2005). La périphrase être en train de, perspective interlinguale (anglais-français) : une modalisation de l'aspect?, in: H. Bat-Zeev Shyldkrot ; N. Le Querler, (éds), Les Périphrases Verbales, Linguisticae Investigationes Supplementa 25, John Benjamins, 119-142.

Leeman, D. (2003). Le passé simple et son co-texte : examen de quelques distributions, Langue Française 138 : 20-34.

Marandin, J.-M. Structure informationnelle, Sémanticlopédie, http:/www.semantique-gdr.net/dico/index.php/Focus. 
Molendijk, A. (1985). Point référentiel et imparfait, Langue Française 67 : 78-94.

Molendijk, A. (1990). Le passé simple et l'imparfait: une approche reichenbachienne, Amsterdam : Rodopi.

Mortier, L. (2005). Les périphrases aspectuelles 'progressives' en français et en néerlandais : présentation et voies de grammaticalisation, in : H. BatZeev Shyldkrot; N. Le Querler, (éds), Les Périphrases Verbales, Linguisticae Investigationes Supplementa 25, John Benjamins, 67-82.

O’Kelly, D. (1995). Temporalité et textualité. Le cas Weinrich, Modèles Linguistiques, 32, XVI, 2 : 145-165.

Pusch, C. D. (2003). La grammaticalisation de l'aspectualité : les périphrases à valeur progressive en français, Verbum XXV-4 : 495-508.

Roussarie, L. Sémanticlopédie, Prédicat, www.semantique-gdr.net/dico/index.php/Accueil.

Tasmowski-De Ryck, L. (1985). L'imparfait avec et sans rupture, Langue Française $67: 58-77$.

Weinrich, H. (1973). Le temps, Paris : Seuil.

Wilmet, M. (1996). L'imparfait : le temps des anaphores ?, Cahiers Chronos $1: 199-215$. 